\title{
CAVIDAD IDIOPÁTICA DE STAFNE: CARACTERÍSTICAS Y CONSIDERACIONES IMAGENOLÓGICAS. UNA REVISIÓN
}

\section{STAFNE'S IDIOPATHIC BONE CAVITY: CHARACTERISTICS AND IMAGENO- LOGICAL CONSIDERATIONS. A REVIEW}

\section{Alexandra Gabriela Cruces Valdivia $*$ (1) \\ ale_cv90@hotmail.com}

\section{Gustavo Adolfo Fiori-Chíncaro² \\ gfiori@ilaeperu.com}

Ana María Agudelo-Botero 3 (1) ortoamariabotero@gmail.com

Artículo recibido: 16/09/2021

Arbitrado por pares

Artículo aceptado: 22/09/2021

Artículo publicado: 26/10/2021

\section{* Autor corresponsal:}

Alexandra Gabriela Cruces Valdivia ale_cv90@hotmail.com

\section{(C) Las autoras, 2021. Publicado por la} Universidad Científica del Sur (Lima, Perú)

Citar como: Cruces-Valdivia AG, FioriChíncaro GA, Agudelo-Botero AM. Cavidad idiopática de Stafne, características y consideraciones imagenológicas. Una revisión. Rev Cient Odontol (Lima). 2021; 9(3): e076.

DOI: 10.21142/2523-2754-0903-2021-076

\section{RESUMEN}

En 1942, el Dr. Edward Stafne presentó una serie de casos con características radiográficas de imágenes radiolúcidas con forma redondeada y bien delimitadas que asemejaban patologías maxilares, que tenían algunos aspectos en común como la ubicación cercana al ángulo mandibular y la relación con la base mandibular, a los que se refirió como defecto óseo. A este defecto se le conocería más adelante con diferentes nombres.

Clínicamente es asintomática, no suele palparse intraoralmente ni presentar signos extraorales. Por ello, en la mayoría de los casos será un hallazgo radiológico accidental, el cual se presenta como una imagen radiolúcida delimitada, elíptica o redondeada, su diámetro varía de 1 a 3 centímetros, aproximadamente, y está delimitada por una osteocondensación en sus límites anteroinferiores. Según su ubicación, es clasificada en tres variantes; anterior, posterior y de rama.

Se debe considerar esta entidad como una variante de normalidad y asociarla con diagnósticos alternativos. Por ello, esta revisión brinda información sobre su historia y sus características generales y radiográficas, con el fin de contribuir a una mejor visión respecto de este tipo de hallazgos.

Palabras clave (MeSH): Stafne, cavidad idiopática de Stafne, cavidad ósea de Stafne, cavidad ósea

\section{ABSTRACT}

In 1942, Dr. Edward Stafne presented 35 cases of asymptomatic, clearly defined, round or ovoid radiolucencies occurring near the angle of the mandible, with a greater incidence below the lower dental canal, between the mandibular angle and the roots of the first lower molar, which he referred to as a bone defect. This bone defect later became known by other names.

Clinically, Stafne's idiopathic bone cavity (SIBC) is asymptomatic, is usually not palpated intraorally and has no present extraoral signs. Thus, in most cases it is an accidental radiological finding, which appears as a delimited, elliptical or rounded radiolucent image, with a diameter ranging from 1 to $3 \mathrm{~cm}$, and delimited by osteocondensation in the anteroinferior limits. According to the location of the findings, they are classified as; anterior, posterior and branch.

This entity should be considered as a variant of normality requiring differential diagnosis to achieve an accurate diagnosis. The present review provides information on the history, and general and radiographic characteristics of SIBC to facilitate diagnosis when presented with this type of findings.

Keywords (MeSH): Stafne, idiopathic Stafne cavity, Stafne bone cavity, bone cavity

\footnotetext{
1 Facultad de Estomatología, Universidad Inca Garcilaso de la Vega. Lima, Perú.

2 Instituto Latinoamericano de Altos Estudios en Estomatología (ILAE). Lima, Perú.

3 Facultad de Estomatología, Universidad Militar Nueva Granada, Fundación Universitaria CIEO. Bogotá, Colombia.
} 


\section{INTRODUCCIÓN}

El Dr. Edward Stafne, en 1942, presentó 35 casos de radiolucidez asintomática bien delimitada, redonda $\mathrm{u}$ ovoide, los cuales se presentaban cerca del ángulo de la mandíbula, con mayor incidencia debajo del conducto dentario inferior, entre el ángulo mandibular y las raíces del primer molar inferior, a las que se refirió como defecto óseo ${ }^{(2-4)}$. A este defecto óseo se le conocería más adelante con diferentes nombres, como cavidad ósea de Stafne, quiste óseo de Stafne, quiste estático, cavidad ósea mandibular, cavidad ósea lingual, concavidad ósea idiopática de la mandíbula, inclusión mandibular de la glándula salival, defecto estático, cavidad ósea latente, cavidad idiopática de hueso, defectos mandibulares de cortical lingual, entre otros $\left({ }^{5,7,17}\right)$.

Clínicamente, la lesión es asintomática, no suele palparse intraoralmente ni presentar signos extraorales $\left({ }^{6,7,15}\right)$; por ello, en la mayoría de los casos, es un hallazgo radiológico accidental. Se presenta como una imagen radiolúcida delimitada, elíptica o redondeada, con un diámetro que varía entre 1 y 3 centímetros, aproximadamente, delimitada por una osteocondensación en sus límites anteroinferiores $(7,13,14)$.

Según su ubicación, es clasificada en tres variantes: anterior, posterior y de rama. La variante anterior se encuentra en el área de la glándula sublingual, la región anterior o el cuerpo de la mandíbula. La variante posterior se encuentra en la zona de la glándula submandibular, que está en la región posterior de la mandíbula. La variante de rama se relaciona con el área de la glándula parótida $\left({ }^{30,31}\right)$. Hay informes en la literatura en los que la cavidad ósea de Stafne tiene variantes que no toman en cuenta dicha clasificación y se presentan en la región anterior de la mandíbula, la rama ascendente de la mandíbula, la región subcondílea, el cuello de la mandíbula, casos simultáneos anterior y posterior, etc. Además, se encuentran casos en los que la cavidad no es única, sino que también puede ser bilobulada, multilocular (presentar varias cavidades) o presentar expansión bucal $(6,12,24,17)$.

A lo largo del tiempo, se consideraron varias patologías para el diagnóstico diferencial: quiste radicular como quiste óseo traumático, ameloblastoma, quiste periapical, queratoquiste ontogénico, quiste óseo simple, lesión central de células gigantes, displasia fibrosa, angioma, mieloma y lesiones fibro-óseas (2,11,25). Para llegar al diagnóstico tenemos la radiografía panorámica, la cual ofrece amplia información. En los casos en los que no se puede dar un diagnóstico definitivo, se utilizan estudios auxiliares como la tomografía computarizada, la resonancia magnética y la sialografía de glándula submandibular $(2,8,15)$.

Por lo expuesto, se debe tomar muy en consideración el defecto óseo de Stafne, tenerlo como referente para proporcionar un diagnóstico certero y así evitar intervenciones innecesarias. Esta revisión de la literatura brindará información sobre su historia, frecuencia, incidencia, características generales y radiográficas, lo que ayudará a lograr una mejor visión con respecto a este tipo de hallazgos.

\section{MATERIALES Y MÉTODOS}

Para la elaboración de este artículo, se llevó a cabo una revisión literaria sistematizada de artículos referentes al tema publicados hasta enero 2021, mediante las bases de datos Medline vía PubMed, SciELO y Scopus. También se consultaron diferentes revistas científicas, como Oral Surgery, Oral Medicine, Oral Pathology and Oral Radiology; Endodontics Journal, Journal Oral Maxillofacial Pathology, International Endodontic Journal, Oral Radiology e Iranian Endodontic Journal. Las palabras claves utilizadas para esta investigación fueron: "Stafne" y "Defecto óseo de Stafne".

\section{Evolución histórica}

E1 defecto óseo que lleva el nombre de Edward Stafne fue descrito por primera vez en 1936, por Louie Austin, quien lo describió como un quiste, el cual no crecía más allá de ciertos límites, debido a que ocurría un drenaje interno que lo encapsulaba y esto hacía que no traspase las superficies óseas; por ello, lo calificó como una patología constante o intermitente $\left({ }^{14}\right)$. 
Posteriormente, el Dr. Edward Stafne, que laboraba en el Departamento de Odontología y Cirugía Oral de la Escuela de Posgrado de Medicina de Mayo, en Rochester (Minnesota, EE. UU.), el 1 de noviembre de 1942, informó sobre 35 casos accidentales con una misma apariencia radiográfica y ubicación, lo cual le hacía pensar que era un factor etiológico común (1,31, 33). Las 35 cavidades se encontraron en 34 pacientes, 17 en el lado derecho y 18 en el izquierdo, en veintiocho hombres y seis mujeres con una edad promedio de 53 años. Los hallazgos se realizaron en películas intraorales y, en algunos casos, extraorales de mandíbula lateral $\left({ }^{1}\right)$.

Las cavidades variaban de 1 a $3 \mathrm{~cm}$ y se encontraban debajo del canal mandibular, delante del ángulo mandibular del tercer molar, y estaban limitadas por hueso esponjoso, y las de mayor tamaño se expandían hasta la superficie del hueso, con una discontinuidad en el borde inferior de la mandíbula, clínicamente se podía sentir mediante palpación. Con respecto a sus características radiográficas, se describe una cavidad de forma circular $\mathrm{u}$ ovaladas, las paredes eran densas y gruesas, diferentes de las producidas por quistes de origen dental revestidas por epitelio. La literatura refiere que Stafne siguió cinco de los defectos durante 5 a 11 años, sin notar cambio alguno en tamaño o características ${ }^{(1)}$.

Más adelante, en 1955, Jacobs realizó la primera exploración quirúrgica del defecto óseo de Stafne, en la que encontró una parte de la glándula submaxilar $\left({ }^{8}\right)$. En 1957 Euclid, Richard y Zikind publican el primer reporte de defecto óseo de Stafne en la zona anterior de un hombre blanco de 46 años. Mediante el examen radiográfico, se encontró una imagen compatible con quiste en la región del canino y el premolar inferior izquierdos, con borde esclerótico bien definido. De acuerdo con el examen clínico, ambos dientes eran vitales. Se realizó la extracción de la lesión el 9 de agosto de 1955 y se observó que el hueso era normal en color y estructura, pero tenía la placa vestibular adelgazada, el tejido se movía libremente, solo había un pequeño pedículo adherido en lingual y el defecto encontrado medía entre 2 y $3 \mathrm{~mm}$ de diámetro. $\mathrm{El}$ examen microscópico reveló una mezcla de estructura glandular compuesta de mucosa y serosa que se asemejaba a los conductos excretores de la glándula salival sublingual. El 9 abril de 1956, se mostró una evidencia de reosificación del área de la lesión $\left({ }^{41}\right)$.

\section{Teorías del origen, epidemiología y clasificación}

La etiología y la patogenia de la depresión o cavidad siguen siendo poco conocidas. La primera teoría defendida por Stafne fue que una porción de la glándula salival es atrapada durante el desarrollo y la osificación de la mandíbula ( $\left.{ }^{32}\right)$. A lo largo del tiempo se han descritos defectos relacionados con las glándulas sublingual y parótida, con distintas localizaciones, y en algunos casos los autores aplican el termino quiste óseo de Stafne a las lesiones de cualquiera de las glándulas salivales $\left({ }^{11}\right)$.

Existen diversas teorías para explicar su origen del defecto óseo de Stafne. La primera la considera un defecto congénito que ocurre durante el desarrollo, cuando la glándula submandibular queda atrapada en la parte posterior de la mandíbula. Stafne planteó que podría ser una malformación ocasionada durante el desarrollo fetal; otros autores señalan que podría deberse a la captura de una porción glandular y la osificación mandibular. Seward dice que lo que mantiene esta hipótesis es la frecuencia en la posición, la similitud en su apariencia, la aparición rara bilateral y los pocos cambios que presenta. La principal discrepancia con esta teoría señala que el defecto es más frecuente en adultos que en niños, lo que demostraría que el desarrollo es más tardío después de la osificación de la mandíbula. A favor de esta teoría se han visto casos en los que su desarrollo es lento $\left({ }^{18-20}\right)$.

La segunda apunta a la presión de la glándula salival adyacente a la superficie interna de la mandíbula. Esta teoría señala que la condición de este defecto proviene de la presión generada por hiperplasia, hipertrofia o desplazamiento de las glándulas salivales hacia la superficie del hueso adyacente cuando están en procesos de formación, lo que ocasiona una remodelación ósea como respuesta. Esto explicaría la presencia del defecto en la zona anterior relacionada con la glándula sublingual, las variables del ángulo mandibular relacionada con la glándula submandibular y las de rama ascendente 
asociadas con las parótidas; sin embargo, esta teoría tampoco daría respuesta de la presencia del defecto en lugares poco comunes $(18,23,24,39)$.

La tercera se relaciona con el tejido blando. En algunos casos, al interior de estas cavidades se encuentra tejido blando que corresponde a vasos, nervios, tejido linfático, grasa y músculos, lo que se debe a la relación entre las estructuras adyacentes a la mandíbula $\left({ }^{8,18}\right)$. La cuarta es la propuesta por Lello y Makek, quienes plantean que las cavidades se producen por la isquemia que ejerce la arteria facial en su trayecto por esta área $\left({ }^{8,18}\right)$.

Ninguna de las teorías expuestas ha sido validada, pero las más aceptadas son las dos primeras, y la explicación es que la primera teoría refiere que el defecto es congénito, es decir, la glándula salival es retenida durante el desarrollo y la osificación. La principal observación es que los defectos se presentan más en adultos que en niños, lo que insinúa que el defecto ocurre después de la osificación; esto con base en casos en los que la cavidad no se presentaba en radiografías panorámicas preliminares. Según Philipsen, esto se justifica porque, con el avance de la edad, las glándulas salivales mayores, y en especial la submandibular, son lugares de infiltración inflamatoria inespecífica (linfocítica) con fibrosis, hipertrofia e hiperplasia, con una intensidad variable. Este proceso varió la consistencia de las glándulas de un tejido normal a uno fibroso. En la edad mediana, la fuerza realizada sobre las estructuras puede ser capaz de ocasionar la reabsorción ósea $\left({ }^{26}\right)$. La segunda teoría aceptada es la que se produce por la presión de la glándula en la superficie adyacente $\left({ }^{28,29}\right)$.

Según su ubicación, se clasifica como: a) Anterior: área de la glándula sublingual, cuerpo de la mandíbula o zona anterior; b) Posterior: zona de glándula submandibular, zona posterior de la mandíbula; c) Rama: área de la glándula parótida en la cual encontramos una variante interna y externa, en algunos casos pueden presentarse en zonas inusuales como en la rama medial, lingual anterior, bilateral, etc. $\left({ }^{8,25,26,31}\right)$.

Ariji et al., en1993, presentaron una clasificación relacionada con la cortical y el contenido. Respecto de la cortical, mencionó tres tipos: Tipo I: se presenta en la cortical lingual; Tipo II: la encontramos en vestibular y lingual de la cortical; Tipo III: agrandamiento de cortical lingual y vestibular $(5,8,35)$. Con relación al contenido, señala otros tres tipos: a) Tipo G: presencia de tejido glandular; b) Tipo F: presencia tejido adiposo; c) Tipo S: Contiene tejido vascular y muscular linfático $\left({ }^{(35,36)}\right.$.

Chaudry, en 2020, menciona limitaciones en la clasificación de Ariji et al. respecto de la cortical, es por eso que presenta una modificación en el sistema existente: Tipo I: la profundidad de la cavidad se limita a la porción medular de la mandíbula; Tipo II: la profundidad de la cavidad llega a la corteza bucal de la mandíbula, pero no provoca su expansión; Tipo IIa: la profundidad de la cavidad llega a la corteza bucal de la mandíbula y provoca su erosión; Tipo IIb: la profundidad de la cavidad llega a la corteza bucal de la mandíbula y provoca su perforación; Tipo III: la profundidad de la cavidad llega a la corteza bucal de la mandíbula y provoca su expansión; Tipo IV: la profundidad de la cavidad llega a la corteza bucal y provoca su expansión y perforación. El autor sugiere que esta modificación es más completa e incorpora todos los TCE linguales; por tanto, ayuda a un mejor seguimiento de estas lesiones $\left({ }^{35,36}\right)$.

Liu et al., por su parte, consideran una clasificación adicional para cuando se emplea la tomografía computarizada de haz cónico, la cual toma en cuenta la cavidad en relación con el conducto mandibular: a) Separado: cavidad que no contacta dicho conducto; b) Contacto: cuando contacta el conducto mandibular; y c) Extendido: la cavidad se extiende por el conducto. También categoriza la relación con respecto a la cortical inferior de la mandíbula en las mismas categorías. Para los casos atípicos, Minowa et al. consideran una clasificación relacionada con las vistas axiales de la tomografía: tipo amplio, tipo estrecho, tipo "margen liso" y tipo "margen irregular" $(34,36,38)$.

Epidemiológicamente la probabilidad de encontrar accidentalmente una cavidad ósea estática en una radiografía panorámica es del $0,08 \%$, y se hallan más en hombres que en mujeres (proporción de 6 a 1), en edades entre los 50 y 70 años, que son las de mayores hallazgos. Sin embargo, se han presentado casos aislados de pacientes pediátricos y en personas adultas mayores 
Tabla 1. Distribución de características de presentación del defecto óseo de Stafne

\begin{tabular}{|c|c|c|}
\hline & NÚMERO & $\%$ \\
\hline \multicolumn{3}{|l|}{ SEXO } \\
\hline Masculino & 79 & $80 \%$ \\
\hline Femenino & 19 & $20 \%$ \\
\hline \multicolumn{3}{|l|}{$\operatorname{EDAD}(*)$} \\
\hline Masculino & 53 & \\
\hline Femenino & 51 & \\
\hline \multicolumn{3}{|l|}{ LADO } \\
\hline Derecha & & $38 \%$ \\
\hline Izquierda & & $54 \%$ \\
\hline Bilateral & & $7 \%$ \\
\hline \multicolumn{3}{|c|}{ LOCALIZACIÓN } \\
\hline Anterior & & $30 \%$ \\
\hline Posterior & & $65 \%$ \\
\hline Rama & & $5 \%$ \\
\hline \multicolumn{3}{|l|}{ FORMA } \\
\hline Redondeada & & $12 \%$ \\
\hline Ovalada & & $87 \%$ \\
\hline Multilocular & & $1 \%$ \\
\hline
\end{tabular}

de 70. La variante lingual posterior se ve con mayor frecuencia en la fosa de la glándula submandibular, con una incidencia del 0,10\% y el 0,48\%. La variante más rara es la de la glándula parótida, que se detalló por primera vez en 1985 y es también llamada variante de rama $(27,28,30,32,34)$ (tabla 1$)$.

\section{Aspectos radiográficos y diagnósticos diferenciales}

El defecto óseo de Stafne es hallado en radiografías de rutina; en la mayoría de los casos no tiene ninguna característica clínica o de sintomatología. Es importante conocer su apariencia radiográfica para emitir un diagnóstico certero y, sea cual sea su presentación, debe ser comparada con patología de origen odontogénico o no odontogénico $\left({ }^{14,31}\right)$.

La apariencia común del defecto óseo de Stafne en radiografía panorámica es la ubicación de una zona radiolúcida - ya sea anterior, posterior o de rama mandibular-, con márgenes bien definidos, de forma ovalada o redonda, y puede ser unilocular o multilocular ${ }^{(26)}$. La más común será una cavidad unilocular definida, la cual está por debajo del canal del nervio dentario inferior o cerca de los ápices de los primeros molares inferiores, con bordes bien definidos y un contenido homogéneo $\left({ }^{14,26}\right)$.

Minowa et al., en 2003, clasificaron la forma de los márgenes de la cortical del defecto óseo en cuatro tipos: amplio, estrecho, liso e irregular $\left({ }^{42}\right)$. Hisatomi et al., en 2019, llevaron a cabo un estudio sobre las características radiográficas de los defectos óseos en radiografías panorámicas, las cuales clasificaron de la siguiente manera: 1) Márgenes: según la presencia de cualquier esclerosis, fina, gruesa o sin esclerosis. Cuando está presente la clasifican como parcial (no aparece en todo el contorno del defecto) o total (aparece en todo el contorno). 2) Grado de radiolucidez interna: definida como parcialmente radiolúcida (presencia de alguna trabécula ósea dentro del defecto) o totalmente radiolúcida (no se observa nada dentro del defecto). 3) Según su forma: ovalada o redonda. 4) Relación topográfica entre el defecto y el borde mandibular: continuidad del defecto hasta la base de la mandíbula o este no toca la base ni la corteza mandibular. 5) Localización del defecto: según su proximidad a los dientes, a excepción de la variante de rama. 6) Locularidad: unilocular o multilocular. 7) Solo para la variante posterior: a) bajo la pared inferior del canal mandibular; b) bajo de la pared superior del canal mandibular y continuo a la pared inferior del canal mandibular; c) debajo de la pared superior del canal mandibular y contiguo a la pared inferior; d) debajo de la pared superior del canal mandibular y superpuesto a la pared inferior del canal mandibular; e) superpuesto a la pared superior e inferior del canal mandibular; $f$ ) continuo con la pared superior del canal mandibular; g) contiguo a la pared del canal mandibular y $\mathrm{h}$ ) por encima del canal mandibular $\left.{ }^{(32}\right)$.

La primera opción para estudiar este defecto óseo será la radiografía panorámica, por ser uno de los primeros estudios auxiliares de rutina y que, por sus características, nos puede ayudar para establecer un diagnóstico. El siguiente examen para tomar en cuenta es la resonancia magnética, que brinda mejor resolución al observar tejidos blandos y tiene ventajas de planos múltiples, 
además de no exponer demasiado al paciente a la radiación ionizante $\left.{ }^{32}\right)$.

La TC es usada como examen auxiliar para dar un diagnóstico certero, ya que sus cortes nos muestra la forma de la cavidad y todas las características en diferentes planos. Existen estudios que afirman que las tomografías computarizadas deben estar respaldadas por resonancias magnéticas, que constituyen un método diagnostico no invasivo, debido a que no emplean radiación ionizante y son usadas en los casos del defecto óseo de Stafne para caracterizar el tejido presente en el defecto sin utilizar medios de contraste $\left({ }^{31,32,34}\right)$.

Otro método diagnóstico es la sialografía, la cual permite evaluar si hay presencia de tejido glandular en la cavidad, pero es un método invasivo para los pacientes y tedioso debido a los múltiples conductos de la glándula $\left({ }^{37}\right)$.

El diagnóstico diferencial para el defecto óseo de Stafne se puede dividir según su formación: neurogenética, histiocítica, vascular o glandular, y resulta de gran ayuda para identificar si se trata de otra patología $\left({ }^{14}\right)$.

\section{CONCLUSIONES}

La cavidad idiopática de Stafne es una condición inofensiva, asintomática, que se encuentra accidentalmente en una radiografía tomada con otro fin diagnóstico. La variante más común es la posterior, que se encuentra por debajo del conducto dentario inferior, entre el ángulo mandibular y las raíces del primer molar inferior, y que suele ser unilocular. Estas características específicas nos permiten establecer un diagnóstico certero sin necesidad de exámenes auxiliares o cirugías exploratorias innecesarias; sin embargo, cuando el aspecto radiográfico de la lesión muestra características diferentes, como imágenes múltiples, multilobuladas y variantes inusuales, deben realizarse exámenes adicionales que ayuden a confirmar o descartar el diagnóstico.

La variante anterior es un hallazgo atípico que puede presentarse en la zona anterior mandibular, así como la variante de rama mandibular. Por eso, es importante que los clínicos conozcan esta variante de la normalidad, así como los protocolos de diagnóstico y seguimiento de estos casos.

Contribución de autoría: Alexandra Gabriela Cruces Valdivia participó en la concepción y el diseño del trabajo, así como en la adquisición y el análisis de los datos, la revisión crítica del manuscrito y la aprobación de su versión final. Gustavo Adolfo Fiori-Chíncaro y Ana María Agudelo-Botero contribuyeron en la concepción y el diseño del trabajo, la revisión crítica del manuscrito y la aprobación de su versión final.

Fuente de financiamiento: Autofinanciado.

Conflicto de intereses: Los autores declaran que no existen conflictos de intereses en relación con la publicación de este artículo. 


\section{REFERENCIAS BIBLIOGRÁFICAS}

1. Stafne EC. Cavidades óseas situadas cerca del ángulo de la mandíbula.J Am Dent Assoc. 1942; 29: 1969-72.

2. Carrillo J, González G, Mastranzo H, Gil G, Reyes S. Defecto óseo en ángulo mandibular (cavidad de Stafne). Presentación de dos casos clínicos y revisión bibliográfica. Revista Mexicana de Cirugía Bucal y Maxilofacial. 2010; 6(2): 73-6.

3. Lee J, Kang S, Jeon S. Stafne bone cavity of the mandible. Arch Craniofac Surg. 22016; 17(3): 162-4. doi: 10.7181/ acfs.2016.17.3.162

4. Segev Y, Puterman M, Bodner L. Stafne bone cavity - Magnetic resonance imaging. Med Oral Patol Oral Cir Bucal 2006; 11: E345-7.

5. Sánchez R, Navarro I, del Castillo Pardo J, Soto M, Burgueño M. Stafne idiopathic bone cavity. Diagnosis and management. Rev Esp Cir Oral Maxilofac. 2011; 33(2): 96-9.

6. Lee K, Thiruchelvam J, McDermott P. An unusual presentation of stafne bone cyst. J. Maxillofac. Oral Surg. 2015; 14(3): 841-4. doi: 10.1007/s12663-014-0737-2

7. Sisman Y, Miloglu O, Sekerci1 AE, Yilmaz AB, Demirtas O, Tokmak ET. Radiographic evaluation on prevalence of Stafne bone defect: a study from two centres in Turkey. Dentomaxillofac Radiol. 2012; 41: 152-8. doi: 10.1259/dmfr/10586700

8. Fernández R, García L, Morales JM, Moyaho A, Montiel A, Loría J. Cavidad ósea idiopática de Stafne: Reporte de un caso y revisión de la literatura. Pren Méd Argent. 2015; 101(7): 386-91.

9. Taysi M, Ozden C, Cankaya B, Olgac V, Yildırım S. Stafne bone defect in the anterior mandible. Dentomaxillofac Radiol 2014; 43: 20140075. doi: 10.1259/dmfr.20140075.

10. Sisman Y, Etoz OA, Mavili E, Sahman H, Tarim E. Anterior Stafne bone defect mimicking a residual cyst: a case report. Dentomaxillofac Radiol. 2010; 39: 124-6. doi: 10.1259/ $\mathrm{dmfr} / 49320253$

11. Branstetter BF, Weissman JL, Kaplan SB. Imaging of a Stafne bone cavity: what MR adds and why a new name is needed. AJNR Am J Neuroradiol. 1999; 20: 587-9.

12. Venkatesh E. Stafne bone cavity and cone-beam computed tomography: a report of two cases.J Korean Assoc Oral Maxillofac Surg. 2015; 41(3): 145-8. doi: 10.5125/jkaoms.2015.41.3.145

13. Grau J, Gay C, Hernández F. Cavidad de Stafne. Revisión a propósito de 6 casos. Avances en Odontoestomatología 1991; 7: 83-7.

14. García L, de la Oz J. Cavidad idiopática de Stafne: Revisión bibliográfica. Intramed Journal 2016; 5(2).

15. Vargas F. Prevalencia del defecto óseo de Stafne evaluado mediante tomografía computarizada de haz cónico [Tesis]. Lima: Universidad San Martín de Porres; 2014.

16. Tiol A, García G, de la O-Zavala J, Rodríguez O. Cavidad ósea de Stafne en un paciente pediátrico: informe de un caso. Revista Odontológica Mexicana. 2018; 22(4).

17. Bihani T, Singh K, Kumar V, Mohapatra S. Solitary radiolucency near the angle of mandible. IP Int J Maxillofac Imaging 2017; 3(4): 124-5. doi: 10.18231/2455-6750.2017.0029
18. Fernández R, García L, Morales J, Moyaho A, Montiel A, Loría J. Cavidad ósea idiopática de Stafne: Reporte de un caso y revisión de la literatura. Prensa Médica Argentina. 2015; 101(7): 386-91.

19. Dagum H, Martínez B, Bruno I. Defecto óseo de Stafne: reporte de 2 casos evaluados mediante CBCT; Anu. Soc. Radiol. Oral Máxilo Facial de Chile 2011; 14: 22-5.

20. Belmonte R, Vélez M, García F, García-Perla A, Pedro A, José, Díaz J, Torres E. A Stafne's cavity with unusual location in the mandibular anterior area. Med Oral Patol Cir Bucal 2005; 10: 173-9.

21. Wadhawan R, Rajan P, Sharma S, Mishra S, Goel N. Stafne cyst in an 80-year-old male: A case report. Int J Res Health Sci 2017; 5(1): 6-9.

22. Dorigatti L, Dias E, da Silva Sampieri M, Ferreira O, Santa'Ana E. Stafne quiste óseo - área radiolúcida en la mandíbula frente al patologías hueso: revisión de la literatura. Rev Cir Traumatol Maxilo-fac, Camaragibe. 2009; 9(3): 35-42.

23. De Almeida C, Mourão B, Menezes A, Miranda A, da Costa E, Ramôa F. Lingual cortical mandibular bone depression: frequency and clinical-radiological features; Brazilian Dental Journal 2013; 24(2): 157-62. doi: https://doi.org/10.1590/01036440201302091

24. Barbosa F, Gallotti S, Araujo D, Martins F, Gotardo J, Valois M, Gomes R, Bezerra T, Falci E. Stafne's bone defect in atypical location: between the subcondylar region and neck of the mandible ramus. Open Journal of Medical Imaging 2017; 7: 1249. doi: 10.4236/ojmi.2017.73012

25. Venkatesh E. Stafne bone cavity and cone-beam computed tomography: a report of two cases. J Korean Assoc Oral Maxillofac Surg. 2015; 41: 145-8.

26. Etöz M, Etöz OA, Sahman H, Sekerci AE, Polat HB. An unusual case of multilocular Stafne bone cavity. Dentomaxillofac Radiol. 2012; 41(1): 75-8. doi: 10.1259/dmfr/34731967.

27. Herranz J . Stafne's bone cavity: An unusual case with involvement of the buccal and lingual mandibular plates. J Clin Exp Dent 2014; 6(1): e96-9. doi: 10.4317/jced.51229

28. Hayashi K, Onda T, Iwasaki T, Takata M, Mori K, Matsuda H, Watanabe S, Tamura H, Shibahara T, Takano M. A case of a Stafne bone defect associated with sublingual glands in the lingual side of the mandible. Case Rep Dent. 2020; 2020: 8851174. doi: 10.1155/2020/8851174

29. Chen MH, Kao CT, Yu-Fong Chang J, Wang YP, Wu YH, Chiang CP. Stafne bone defect of the molar region of the mandible. J Dent Sci. 2019 Dec; 14(4): 378-82. doi: 10.1016/j. jds.2019.05.002

30. He J, Wang J, Hu Y, Liu W. Diagnosis and management of Stafne bone cavity with emphasis on unusual contents and location. J Dent Sci. 2019 Dec; 14(4): 435-9. doi: 10.1016/j. jds.2019.06.001

31. More CB, Das S, Gupta S, Patel P, Saha N. Stafne's bone cavity: a diagnostic challenge. J Clin Diagn Res. 2015 Nov; 9(11): ZD169. doi: $10.7860 / J C D R / 2015 / 14273.6772$ 
32. Hisatomi M, Munhoz L, Asaumi J, Arita ES. Parotid mandibular bone defect: A case report emphasizing imaging features in plain radiographs and magnetic resonance imaging. Imaging Sci Dent. 2017 Dec; 47(4): 269-73. doi: 10.5624/isd.2017.47.4.269

33. Schneider T, Filo K, Stadlinger B, Locher M, Kruse L. Inusual cavidad Stafne. Swiss Dent J. 2014; 124(5).

34. Hernández-Andara A, Ortega-Pertuz A, Saavedra A, Gómez M, Villarroel-Dorrego M. Presentación inusual de cavidad ósea de Stafne: estudio mediante tomografía computarizada y resonancia magnética. Odontología Sanmarquina 2019; 22(4): 299-306.

35. Ariji E, Fujiwara _N, Tabata O, Nakayama E, Kanda S, Shiratsuchi Y, Oka M. Stafne's bone cavity. Classification based on outline and content determined by computed tomography. Oral Surg Oral Med Oral Pathol. 1993 Sep; 76(3): 375-80. doi: 10.1016/0030-4220(93)902715

36. Chaudhry A. Defecto óseo de Stafne con perforación bicortical: necesidad de un sistema de clasificación modificado. Radiología Oral 2021. doi: 10.1007/s11282-020-00457-8

37. Sisman Y, Etöz OA, Mavil E, Sahman H, Tarim Ertas E. Anterior Stafne bone defect mimicking a residual cyst: a case report. Dentomaxillofac Radiol. 2010; 39(2): 124-6.

38. Ehmet-Zahit A, Yilmaz S, Misirlioglu M, Atil F. Evaluation of volumetric measurements on CBCT images using Stafne bone cavities as an example. Journal Med Oral Patol Oral Cir Bucal. 2015 Sep 1; 20(5): e580-6.
39. Chen A, Ahn Y, Odell S, Mupparapu M, Graham DM. Depresión del desarrollo de las glándulas salivales en la rama mandibular ascendente: estudio de tomografía computarizada de haz cónico. Ciencias de la Imagen en Odontología 2016; 46: 223-7.

40. Hisatomi M, Munhoz L, Asaumi J, Arita ES. Características radiográficas de defectos óseos de Stafne en radiografías panorámicas: valoración de 91 casos. Med Oral Patol Oral Cir Bucal. 2019; 24(1): e12-9. doi: 10.4317/medoral.22592

41. Richard EL, Zikind J. Aberrant salivary gland tissue in mandible. Oral Surg Oral Med Oral Pathol. 1957 Oct; 10(10): 1086-90. doi: 10.1016/0030-4220(57)90059-2

42. Minowa K, Inoue N, Sawamura T, Matsuda A, Totsuka Y, Nakamura M. Evaluation of static bone cavities with CT and MRI. Dentomaxillofac Radiol. 2003, 32(1): 2-7. doi: 10.1259/ $\mathrm{dmfr} / 92201340$

43. Aps JKM, Koelmeyer N, Yaqub C. Stafne's bone cyst revisited and renamed: the benign mandibular concavity. Dentomaxillofac Radiol. 2020 May 1; 49(4): 20190475. doi: 10.1259/ dmfr.20190475. Epub 2020 Feb 3. PMID: 31944858; PMCID: PMC7213533 